\title{
From Wendelstein 7-X to a Stellarator Reactor
}

\author{
Robert C. WOLF, Craig D. BEIDLER, Rainer BURHENN, Joachim GEIGER, Matthias HIRSCH, \\ Johann KISSLINGER, Henning MAAßBERG, Arthur WELLER, Andreas WERNER \\ and the Wendelstein 7-X Team \\ Max-Planck-Institut für Plasmaphysik, IPP-EURATOM Association, D-17491 Greifswald, Germany
}

(Received 9 January 2009 / Accepted 2 August 2009)

\begin{abstract}
Wendelstein 7-X is a drift-optimized stellarator with improved thermal and fast ion confinement. Additional optimization criteria are a stiff equilibrium configuration and MHD stability up to a volume averaged $\beta$ of $5 \%$. The main objectives are to demonstrate reactor-relevant plasma performance under steady-state conditions including power and particle exhaust with an island divertor. To that effect Wendelstein 7-X has superconducting coils with a maximum average magnetic field of $3 \mathrm{~T}$ and will be equipped with actively cooled plasma facing components for heat fluxes of up to $10 \mathrm{MW} / \mathrm{m}^{2}$. Besides fulfilling this research mission, the extrapolation from Wendelstein 7-X to a stellarator reactor is an important issue. The capability of such an extrapolation will depend also on the results from other stellarators, on the ITER results and here in particular the experience gained with $\alpha$-particle heating and operating a nuclear device, and the advancement of first-principle theory required for the extrapolation.
\end{abstract}

(c) 2010 The Japan Society of Plasma Science and Nuclear Fusion Research

Keywords: Wendelstein 7-X, stellarator optimization, HELIAS, steady-state operation

DOI: $10.1585 /$ pfr.5.S1011

\section{Introduction}

Wendelstein 7-X (W7-X) is a drift-optimized low magnetic shear stellarator designed to demonstrate the basic reactor capability of the stellarator concept [1]. With a major radius, $R$, of $5.5 \mathrm{~m}$ and an average minor radius, $<a>$, of $0.5 \mathrm{~m}$ the resulting plasma volume of $30 \mathrm{~m}^{3}$ lies between those of ASDEX Upgrade and JET. The maximum magnetic field is $3 \mathrm{~T}$, corresponding to $600 \mathrm{MJ}$ of magnetic field energy. The rotational transform, $t$, ranges from $5 / 6$ to $5 / 4$ and, in contrast to the partially optimized predecessor of W7-X, Wendelstein 7-AS (W7-AS) [2], is practically independent of the plasma $\beta$.

The design of $\mathrm{W} 7-\mathrm{X}$ is based on an elaborate optimization procedure to overcome the essential deficiencies of the stellarator concept: (1) The introduction of quasisymmetry and quasi-isodynamicity - in case of W7-X the latter was used - yields reduced neoclassical transport and, in particular, good fast ion confinement which is a prerequisite for any type of fusion reactor. Since in a stellarator the neoclassical diffusion scales like $\varepsilon_{\mathrm{eff}}{ }^{3 / 2} T^{7 / 2}$, the effective ripple, $\varepsilon_{\text {eff }}$ [3], has to be kept as small as possible. In addition, to keep the temperature low sufficiently high density is required. (2) By minimizing the Pfirsch-Schlüter currents the Shafranov shift is minimized and thus a high equilibrium limit is achieved. (3) The minimization of the bootstrap current serves several purposes. First, it removes free energy necessary for current driven instabilities. Secondly, it helps to avoid low order rational values in the low-shear $t$-profile which have negative

author'se-mail: robert.wolf@ipp.mpg.de impacts on confinement and stability properties. Thirdly, a considerable amount of bootstrap current would severely influence the operation of an island divertor. (4) Finally, the magnetic field configuration provides sufficiently large magnetic well to avoid pressure-driven instabilities such as interchange modes, aiming at a volume-averaged $<\beta>$ of $5 \%$.

High-power steady-state operation of W7-X will be approached in two steps: (1) An inertially cooled test divertor will allow pulses from 10 to $50 \mathrm{~s}$, corresponding to heating power levels from 8 to $11 \mathrm{MW}$. During this initial phase three heating systems will be available: Neutral beam injection (NBI), ion cyclotron resonance heating (ICRH) and electron cyclotron resonance heating (ECRH). Depending on the combination of the heating methods, the available heating power will vary between 8 and $11 \mathrm{MW}$. (2) After the installation of the actively cooled divertor and the completion of the water cooling of all plasma facing components, 30 minutes plasmas with $10 \mathrm{MW}$ ECRH [4] are foreseen. Later upgrades will include increases of the neutral beam heating power from 10 to $20 \mathrm{MW}$ and of the ion cyclotron heating power from 2 to $10 \mathrm{MW}$.

Various studies of a HELIcal Advanced Stellarator (HELIAS) reactor have been conducted already (see e.g. $[5,6])$. The HELIAS reactor is basically an extrapolation from the W7-X design, which in itself is based on results from the W7-AS stellarator, the first advanced stellarator experiment. Essentially, three requirements form the basis of the HELIAS reactor concept: (1) Sufficiently good confinement has to be guaranteed to reach ignition. 
Probably owing to the different weighting of neoclassical and anomalous transport in the different stellarator devices, a unified scaling law does not exist. Recent studies have shown that the cross-device scaling improves with the introduction of a configuration factor, which appears to correlate with the degree of neoclassical optimization [7]. Therefore, conservatively, a confinement improvement, such as observed in the H-mode, has not been assumed. (2) To provide a superconductor with sufficient ductility for the fabrication of the non-planar modular coils, NbTi has been chosen. At the temperature of superfluid helium of $1.8 \mathrm{~K}$ maximum magnetic fields of $10 \mathrm{~T}$ are possible, corresponding to $5 \mathrm{~T}$ on axis. (3) For the blanket a space of $1.3 \mathrm{~m}$ thickness has been reserved between plasma and coils. Although no detailed design for the blanket has been made up to now, this is thought to be sufficient. With $\langle\beta\rangle=4-5 \%$, the resulting HELIAS reactor concepts have $3 \mathrm{GW}$ of fusion power. Three types with different aspect ratios, but similar plasma volumes, have been investigated [8]: The HR5/22 with a five-fold symmetry, a major radius of $R=22 \mathrm{~m}$, an aspect ratio of $A=12$ and 50 modular coils, the HR $4 / 18$ with $R=18 \mathrm{~m}, A=9$ and 40 modular coils, and the HR $3 / 15$ with $R=15 \mathrm{~m}, A=6$ and 30 modular coils. Less coils for a given plasma volume means lower cost. However, the limit for reducing the aspect ratio is given by the $\alpha$-particle losses, which for the HR3/15 already become critically high. Therefore, the HR4/18 is regarded as the best compromise between a reasonably small number of coils and sufficient $\alpha$-confinement. Because of the low volume to surface ratio, the advantage of a large aspect ratio is a low neutron flux to the wall which for the HR4/18 is calculated to be on average $1 \mathrm{MW} / \mathrm{m}^{2}$ reaching peak values of $1.6 \mathrm{MW} / \mathrm{m}^{2}$.

Recently the European fusion facilities have undergone an extensive review to assess their relevance for the future fusion programme. Seven R\&D missions have been defined which provide an efficient and focused implementation of the fusion programme [9]. Their topics are burning plasma, reliable operation, operation compatible with first wall, technology and physics for steady-state operation, predicting fusion performance, operation in nuclear environment and DEMO integrated design. The following discussion of the W7-X programme will be made with respect to these R\&D missions. The last two missions, however, do not apply, as W7-X is neither a nuclear device (no deuterium-tritium operation) nor will DEMO components such as a breeder blanket be tested in W7-X.

\section{Burning Plasma}

The confinement of the fast helium ions or $\alpha$-particles from the D-T fusion reactor is a prerequisite for a future fusion reactor. While in tokamaks it has been demonstrated that at least for low fast ion pressure $\alpha$-particles are confined [10] and heat the plasma [11], in stellarators this is not so easily achieved. Without optimization
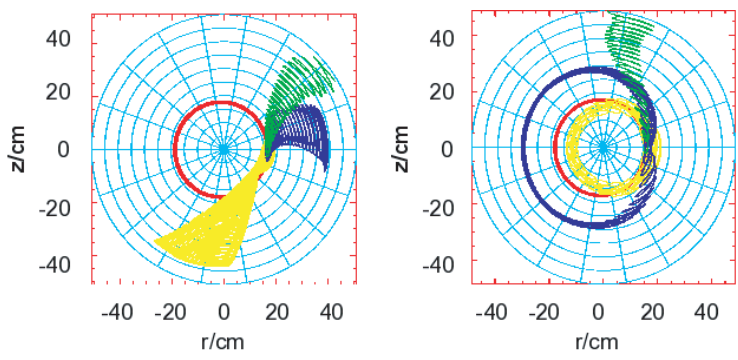

Fig. 1 Poloidal projection of $50 \mathrm{keV}$ proton guiding centre orbits at $2.5 \mathrm{~T}$ in the $\mathrm{W} 7-\mathrm{X}$ high-mirror configuration. Left: vacuum field. Right: $\langle\beta\rangle=2.5 \%$. The colours denote the pitch angles: $60^{\circ}$ (red), $70^{\circ}$ (yellow), $75^{\circ}$ (blue), $80^{\circ}$ (green). $60^{\circ}$ is a passing orbit, the others are trapped ones.

of the stellarator magnetic field configuration, in the long mean-free-path regime fast ions tend to drift radially and thus leave the confinement region. The quasi-isodynamic property of W7-X, which appears at finite $\beta$, utilizes the diamagnetic effect to increase the poloidal drift velocity of deeply trapped particles to make them precess poloidally. This, however, also relies on reduced radial grad- $B$ drift velocities (drift-optimization), which is realized in W7-X in a field configuration with a strong toroidal mirror field and a proper ratio of toroidal and helical field harmonics. With the five field periods, this roughly establishes a system of five linked mirrors [12]. The trapped particles oscillate between the regions of high magnetic field, undergoing a net poloidal rotation but little net radial movement as they are kept away from zones of high field inhomogeneity (responsible for large radial drift velocities). Fig. 1 shows the orbits of $50 \mathrm{keV}$ protons calculated for the high-mirror configuration of W7-X [13] (magnetic field of $2.5 \mathrm{~T}$ ) for vacuum and for a $<\beta>$-value of $2.5 \%\left(\beta_{0} /<\beta>=3\right)$. It can be seen that trapped fast ions are not confined in the vacuum field, but for finite- $\beta$ they are forced on poloidal orbits (poloidal transit times for $70^{\circ}$ and $75^{\circ}$ are $0.4 \mathrm{~ms}$ and $0.7 \mathrm{~ms}$, respectively). Prompt losses occur on a time scale of $0.1 \mathrm{~ms}$ for $50 \mathrm{keV} \mathrm{H}^{+}$ions in $\mathrm{W} 7-\mathrm{X}$ as well as for 3.5 MeV $\alpha$-particles in a reactor, while the energy slowing down times are $\approx 20 \mathrm{~ms}$ for $\mathrm{W} 7-\mathrm{X}$ and $\approx 100 \mathrm{~ms}$ for the reactor, given the expected $(n, T)$-values.

A $<\beta>$ of $2.5 \%$ is not sufficient to confine all trapped particles, but must be increased to nearly $5 \%$ to achieve this [14]. This means that the full demonstration of fast ion confinement will be possible only during the second phase of operation, as power and pulse duration are limited during the first phase. To reach a fully equilibrated magnetic field configuration the pulse duration has to be of the order of some $L / R$-times ( $L$ and $R$ are plasma inductance and resistance, respectively). The estimated $L / R$-time is about 20-40 s for W7-X parameters.

Extrapolated to the HELIAS reactor (HR4/18) a $\alpha$ loss fraction of $2.5 \%$ is predicted. With respect to the power balance this is in any case not critical, but in a fusion reactor the $\alpha$-losses have to be kept this low, as undue lo- 
calized fast ion fluxes leaving the plasma will damage first wall components.

\section{Reliable Operation}

To achieve a high fusion power density, a fusion reactor requires high $\beta$ and high plasma density. This, however means, that reliable operation near operational boundaries will be needed. Here, stellarators have a clear advantage as, without current driven instabilities and disruptions, the plasma behaviour at the $\beta$-limit is fairly benign. In addition, much higher densities can be achieved in stellarators than in tokamaks, as the Greenwald limit, known from tokamaks, has not been observed $[2,15,16]$.

While W7-AS still showed pressure driven modes, the W7-X design should include sufficient magnetic well to provide stability up to $<\beta>=5 \%$, at least for the standard and high mirror configurations. However, also in W7-AS examples exist where the increase of $\langle\beta>$ eventually led to a stabilization of these modes. This is explained by the deepening of a magnetic well and the formation of magnetic shear as the configuration changes with rising $\langle\beta>$. In high- $\beta$ discharges at reduced magnetic field the vertical field had to be increased to compensate for the larger Shafranov shift in such a way that the magnetic well almost disappeared in the vacuum configuration. In such cases the magnetic well even reformed when $\beta$ was increased. While in W7-AS, again at reduced field, the equilibrium limit has been reached [17], in W7-X, owing to a small Shafranov shift, also the equilibrium limit should not curtail the value of $\langle\beta>=5 \%$.

Already during the $1^{\text {st }}$ operational phase high density will be addressed. To this effect $\mathrm{W} 7-\mathrm{X}$ is equipped with an ECRH system prepared for $2^{\text {nd }}$ harmonic O-mode which works above electron densities of $n_{\mathrm{e}}=1 \times 10^{20} \mathrm{~m}^{-3}$. Based on neoclassical transport in the plasma core (and an anomalous edge) at a density of $1.8 \times 10^{20} \mathrm{~m}^{-3}$ and $10 \mathrm{MW}$ of ECRH, electron and ion temperatures of $T_{\mathrm{e}}=6.2 \mathrm{keV}$ and $T_{\mathrm{i}}=4.2 \mathrm{keV}$, corresponding to $\langle\beta>=4.1 \%$, are predicted. Depending on the actual confinement, high $\beta$ might be possible only at lower magnetic field (meaning below $2.5 \mathrm{~T}$, which is the nominal field for $140 \mathrm{GHz}$ ECRH). During the $2^{\text {nd }}$ phase of operation $\beta$ - and equilibrium limit studies will become possible at power levels of $20 \mathrm{MW}$ and above.

With $<\beta>=4-5 \%$ and $n_{\mathrm{e}}=2-3 \times 10^{20} \mathrm{~m}^{-3}$ the HELIAS does not exceed the values envisaged for W7-X. Confinement time (1.6-2.3 $\mathrm{s})$ and ion temperature $\left(T_{\mathrm{i}}=11\right.$ $15 \mathrm{keV}$ ) are of course higher.

\section{Operation Compatible with First Wall}

On the one hand, an undue contamination of plasma with impurities from the first wall has to be avoided and, on the other hand, wall erosion and tritium retention have to be limited. Therefore, carbon is ruled out as a first wall

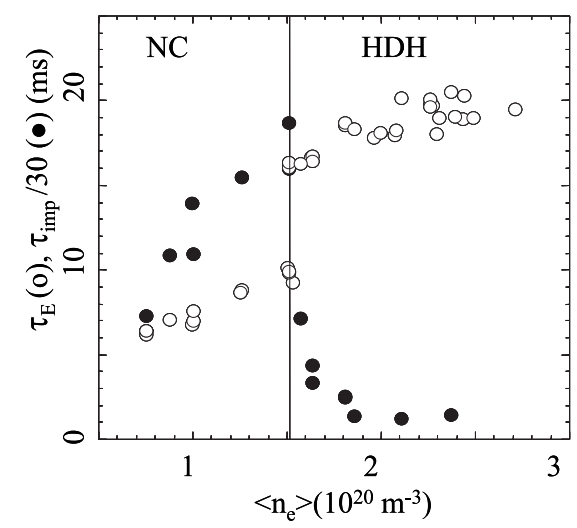

Fig. 2 Energy and impurity confinement time as a function of plasma density. The transition from normal confinement, $\mathrm{NC}$, to the HDH-mode becomes evident in a rise of $\tau_{\mathrm{E}}$ and a simultaneous drop of $\tau_{\text {imp }}$ (decay time of injected aluminum ions in the highest ionization states being a measure for the aluminum ion confinement time).

material and tungsten is considered as a candidate material for a fusion reactor [18].

Nevertheless, W7-X will start operation with carbon covering the high heat flux target elements $\left(\geq 1 \mathrm{MW} / \mathrm{m}^{2}\right)$. For stellarators in particular impurity accumulation is a critical issue. In contrast to tokamaks where the "ion temperature screening" of impurity ions is a beneficial mechanism counteracting impurity accumulation, in stellarators all thermodynamic forces are predicted to support accumulation in the standard case with negative radial electric field, the so-called ion-root regime [19]. For the power and particle exhaust W7-X is equipped with an island divertor which utilizes the large magnetic islands forming at the plasma boundary at $t=1$. This concept was for the first time successfully tested in W7-AS. In W7-AS the introduction of the island divertor also led to the discovery of the high density H-mode (HDH-mode), which not only showed improved energy confinement, but at the same time much reduced impurity confinement without impurity accumulation [20]. This very favourable behaviour is illustrated in Fig. 2. The explanation for the low impurity confinement in HDH is a reduced inward convection in the core (going along with a flattening of the ion density profile) and possibly a mechanism of enhanced outward transport in the density gradient region. The latter, however, has not yet been understood. Additionally, the impurity influx has been effectively screened by the onset of friction forces in the high collisionality scrape-off-layer region [21]. Because of these properties the HDH-mode is the candidate scenario for high density steady-state plasma operation in W7-X. However it is unclear how the HDH scales to W7-X.

During the $1^{\text {st }}$ phase of $\mathrm{W} 7-\mathrm{X}$ operation the divertor topology will have to be investigated. Here, the inertially cooled test divertor has the advantage that, because of its intrinsically robust design, overheating or damaging the 


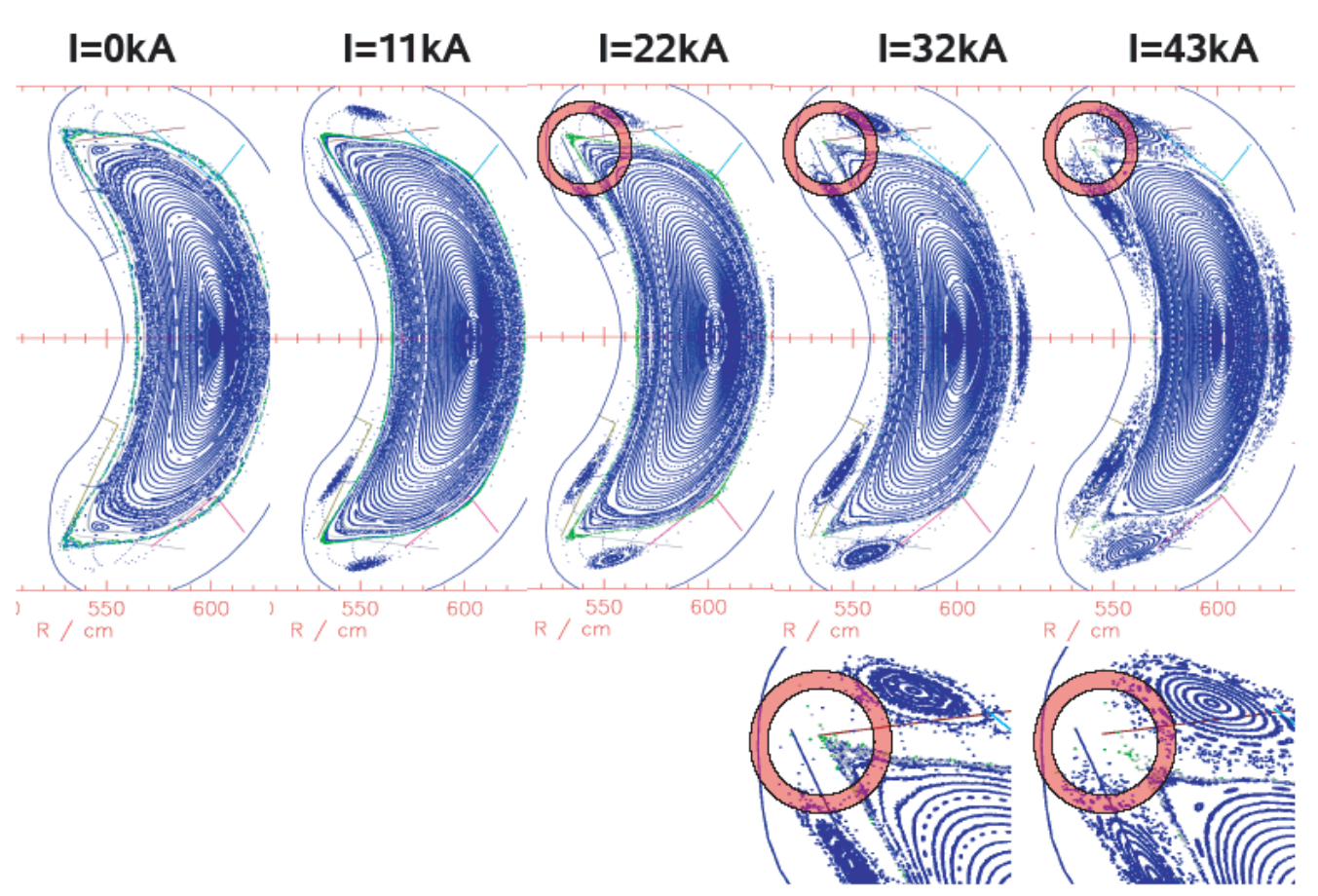

Fig. 3 Sequence of W7-X equilibria with different amounts of plasma currents simulating the current diffusion while a bootstrap current of $43 \mathrm{kA}$ is building up. The red circles show the critical regions near the edges of the divertor tiles which for the last two equilibria at 32 and $43 \mathrm{kA}$ are scaled up.

cooling structure does not have to be considered. Subsequently, first attempts to re-establish the HDH-mode will be made. The $2^{\text {nd }}$ phase of operation will then address full steady-state power and particle exhaust. Eventually also high- $Z$ wall materials, such as tungsten, will have to be considered. Here, a gradual increase of the wall coverage as successfully demonstrated in ASDEX Upgrade [22] could be a feasible approach.

\section{Technology and Physics for Steady State}

$\mathrm{W} 7-\mathrm{X}$ is the first optimized stellarator with an integrated design for steady-state operation. This includes superconducting coils made of $\mathrm{NbTi}$ for a magnetic field strength of up to $3 \mathrm{~T}$ on axis, an actively cooled first wall for heat fluxes of up to $10 \mathrm{MW} / \mathrm{m}^{2}$ [23], and device control, data acquisition, diagnostics and an ECRH system [4] developed for continuous plasma operation. Many aspects of these technologies are similar to the ITER requirements.

During the $1^{\text {st }}$ operational phase of $\mathrm{W} 7-\mathrm{X}$, stationary plasma operation is only possible at very low power levels of the order of $100 \mathrm{~kW}$ to test basic system properties. Going to $1 \mathrm{MW}, 50 \mathrm{~s}$ pulses will already give some insight into plasma behaviour for times longer than the $L / R$ time. In addition to what is described in the previous chapters, short pulses ( $8 \mathrm{MW}$ for $\sim 10 \mathrm{~s}$ ) will be used to verify the improved neoclassical transport. In the $2^{\text {nd }}$ phase the objective is to develop a fully steady-state high-power plasma scenario demonstrating the reactor capability of the stel- larator.

The plasma control requirements of $\mathrm{W} 7-\mathrm{X}$ are generally very low. This also applies to the necessity to control the magnetic field. One of the optimization criteria of the W7-X design is the minimization of the bootstrap current. While the smallest bootstrap current is expected in the high-mirror configuration [13], calculations show that in the standard configuration [13], depending on confinement and density, bootstrap currents of up to $50 \mathrm{kA}$ could develop at $5 \mathrm{MW}$ heating power. Assuming neoclassical confinement in the plasma core the bootstrap current ranges from approximately $45 \mathrm{kA}$ at $4 \times 10^{19} \mathrm{~m}^{-3}$ to about $10 \mathrm{kA}$ at $2 \times 10^{20} \mathrm{~m}^{-3}$. As a consequence of the bootstrap current the plasma equilibrium will show an initial temporal evolution. To illustrate this effect, a sequence of equilibria with different amounts of toroidal currents have been calculated using the VMEC code and a field extender for the magnetic island regions outside the last closed flux surface (Fig. 3). The current diffusion is simulated by assuming that according to Lenz's rule the bootstrap current, $I_{\mathrm{BS}}$, is initially balanced by an "ohmic" current, $\int \sigma E \mathrm{~d} A(E$ : toroidal electric field, $\int \mathrm{d} A$ : integral over poloidal crosssection), where the electrical conductivity, $\sigma$, is essentially given by the temperature distribution. The "ohmic" current is then successively reduced, until finally the total current, $I=I_{\mathrm{BS}}+\int \sigma E \mathrm{~d} A$ matches the bootstrap current. Fig. 3 shows an example where the final equilibrium with the fully developed plasma current is consistent with the island divertor operation. However, on the way to this configuration, the edges of the divertor tiles and the pumping gap 
will be loaded with plasma. To avoid high thermal loads at these regions, either current drive must be applied simultaneously with ECRH or the rotational transform has to be adjusted, since $\mathrm{W} 7-\mathrm{X}$ is not equipped with an ohmic transformer. In W7-X, changes of $t$ during plasma operation should be in principle possible by using the planar coils, but is technically limited due to a limited number of allowed load cycles of the support structure of the magnets. An additional option is the introduction of protection limiters which during the described transition phase take up the heat load from the plasma.

\section{Predicting Fusion Performance}

The prime objective of $\mathrm{W} 7-\mathrm{X}$ is to demonstrate the basic reactor capability of the stellarator. $\mathrm{W} 7-\mathrm{X}$ will have to verify the theory-based optimization criteria and thereby also improve the theoretical understanding of stellarator physics. In a more general context, there are however also a range of physics issues related to $3 \mathrm{D}$ effects concerning both stellarators and tokamaks. E.g., in stellarators the generation of ambipolar electric fields is caused by the open magnetic field lines in the plasma boundary. Recently, the effect of such electric fields on momentum transport has been observed in tokamaks with an ergodic divertor [24]. The control of edge localized modes (ELMs) using dedicated perturbation coils to ergodize the plasma edge is now one of the methods to mitigate ELMs in ITER. In this context W7-X could provide information on ELM control without strong plasma currents and, thus, without the contribution of current-driven instabilities. As an example Fig. 4 shows how the ELM phenomenology in W7-AS is sensitively modified by varying the edge rotational transform over one of the "iota-windows" where a quiescent $\mathrm{H}$-mode is achieved [2]. With parameters close to a transition to the quiescent $\mathrm{H}$-mode regular ELMs with

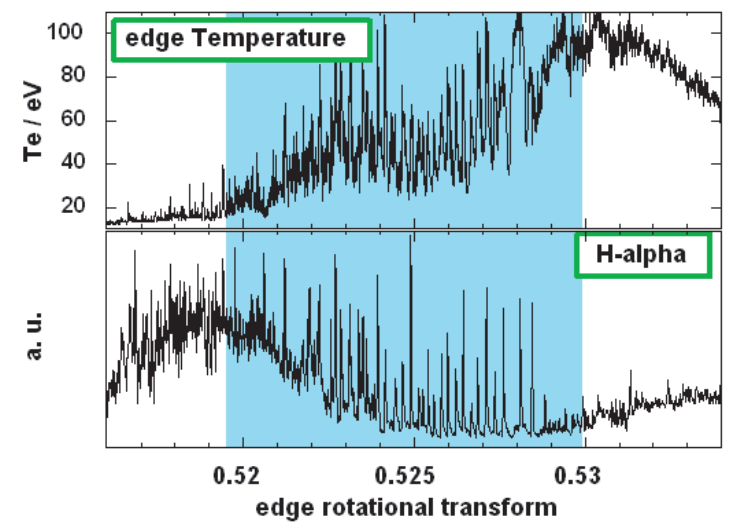

Fig. 4 W7-AS ELM phenomenology ( $T_{\mathrm{e}}$ from ECE measured 1 to $2 \mathrm{~cm}$ inside the separatrix and $\mathrm{H} \alpha$ - monitor) changing while the edge-t is scanned across an $\mathrm{H}$-mode window during $150 \mathrm{~ms}$. Line average density and heating power are kept constant at values right below the conditions where within the blue shaded $t$-window a quiescent H-mode occurs [2]. expressed spikes in $\mathrm{H} \alpha$ and deep crashes in the edge temperature occur. ELM mitigation is possible by slightly tuning the edge $-t$. The states with regular ELMs can be maintained stationary by feedback controlling the density at a fixed magnetic configuration.

The size of the step from W7-X to a larger stellarator device towards a stellarator reactor will depend on the ability to extrapolate the results. This includes the transferability of the ITER results to stellarators, and here in particular the $\alpha$-particle heating, and will strongly depend on the progress made with first principle theories and their applicability to $3 \mathrm{D}$ magnetic field configurations. In this context Wendelstein 7-X fits well into the ITER schedule and the development plan for high performance computing.

\section{Summary and Conclusions}

Wendelstein 7-X is an optimized stellarator designed for steady state operation. Its main objective is the demonstration of the basic reactor capability of the stellarator.

W7-X addresses the main physics issues for the development of a stellarator reactor: (1) Fast particle confinement and fast particle driven instabilities in a 3D configuration: Owing to the high plasma densities, for the latter a reduced drive for Alfvén instabilities is expected. (2) Neoclassical versus turbulent transport: Latest calculations suggest also a dependence of the turbulent transport on the degree of neoclassical optimization [25-27]. (3) Impurity confinement: Plasma scenarios, such as the HDH-mode have to be further developed to avoid impurity accumulation. Also here the possible role of a turbulent drive has to be investigated. In fact, a power degradation of the impurity confinement has been observed in many stellarators [28]. (4) 3D divertor configuration: W7-X will utilize the natural magnetic islands at the plasma boundary and combine them with actively cooled divertor targets. The resonant island divertor relies on a small bootstrap current to avoid undue changes of the edge- $t$ with increasing $\beta$.

Correspondingly specific stellarator technology issues are: (1) Coil configuration, coil support structure and the choice of superconductor. In a stellarator with modular non-planar coils the forces on the support structure need particular attention. (2) Divertor. In a 3D magnetic field configuration a divertor is also technically more complicated, requiring very accurate alignment to avoid unbalanced heat load distributions. (3) Depending on the aspect ratio, the possibility to provide enough space can be very limited in stellarators. (4) In addition, both accessibility and maintainability need to be thoroughly investigated in future stellarator reactor studies.

The further optimization of stellarators [29] has to include also simplified engineering solutions. In this respect, a first-of-a-kind device such as W7-X is not optimized. Future stellarator reactor studies will have to combine physics and engineering optimization in a more rigorous way. 
[1] G. Grieger et al., Phys. Fluids B4, 2081 (1992).

[2] M. Hirsch et al., Plasma Phys. Control. Fusion 50, 053001 (2008).

[3] V. V. Nemov, S. V. Kasilov, W. Kernbichler and M. F. Heyn, Phys. Plasmas 6, 4622 (1999).

[4] M. Thumm et al., Proc. $18^{\text {th }}$ Int. Toki Conf.

[5] H. Wobig, Plasma Phys. Control. Fusion 41, A159 (1999).

[6] C. D. Beidler et al., Nucl. Fusion 41, 1759 (2001).

[7] A. Dinklage et al., Fusion Sci. Technol. 51, 1 (2007).

[8] Yu. Igitkhanov et al., Fusion Eng. Des. 81, 2695 (2006).

[9] J. Pamela, private communication.

[10] M. J. Mantsinen et al., Phys. Rev. Lett. 88, 105002 (2002).

[11] P. R. Thomas et al., Phys. Rev. Lett. 80, 5548 (1998).

[12] E. Strumberger, Nucl. Fusion 40, 1697 (2000).

[13] T. Andreeva. J. Kisslinger and H. Wobig, Problems of Atomic Science and Technology 2002, No.4, Series: Plasma Physics (7), P. 45-47.

[14] W. Lotz et al., Plasma Phys. Control Fusion 34, 1037 (1992).
[15] T. Morisaki et al., Phys. Plasmas 14, 056113 (2007).

[16] M. Greenwald, Plasma Phys. Control. Fusion 44, R27 (2002).

[17] A. Reiman et al., Nucl. Fusion 47, 572 (2007).

[18] M. Kaufmann and R. Neu, Fusion Eng. Des. 82, 521 (2007).

[19] H. Maßßberg, C. D. Beidler, E. E. Simmet, Plasma Phys. Control. Fusion 41, 1135 (1999).

[20] K. McCormick et al., Phys. Rev. Lett. 89, 015001 (2002).

[21] Y. Feng et al., Nucl. Fusion 46, 807 (2006).

[22] R. Neu, J. Nucl. Mater. 363-365, 52 (2007).

[23] R. Stadler et al., Proc. $18^{\text {th }}$ Int. Toki Conf.

[24] K.-H. Finken et al., Phys. Rev. Lett. 94, 015003 (2005).

[25] H. E. Mynick and A. H. Boozer, Proc. $17^{\text {th }}$ Int. Toki Conf.

[26] H. Sugama, T.-H. Watanabe and S. Ferrando-Margalet, Proc. $17^{\text {th }}$ Int. Toki Conf.

[27] F. Jenko et al., Proc. $18^{\text {th }}$ Int. Toki Conf.

[28] R. Burhenn et al., Nucl. Fusion 49, 065005 (2009).

[29] A. A. Subbotin et al., Nucl. Fusion 46, 921 (2006). 